\title{
Carnets
}

Revue électronique d'études françaises de l'APEF

Deuxième série - 22 | 2021

Sous le double signe de Baudelaire et de Flaubert: traductions, adaptations, transpositions

\section{Arthur Symons traducteur de Baudelaire : D'une traduction partielle à une traduction intégrale}

\section{Raphaël Pittier}

\section{(2) OpenEdition}

Journals

Édition électronique

URL : https://journals.openedition.org/carnets/13032

DOI : $10.4000 /$ carnets. 13032

ISSN : 1646-7698

Éditeur

APEF

Référence électronique

Raphaël Pittier, « Arthur Symons traducteur de Baudelaire : D'une traduction partielle à une traduction intégrale », Carnets [En ligne], Deuxième série - 22 | 2021, mis en ligne le 30 novembre 2021, consulté le 30 mai 2022. URL : http://journals.openedition.org/carnets/13032 ; DOI : https://doi.org/10.4000/ carnets. 13032

Ce document a été généré automatiquement le 30 mai 2022.

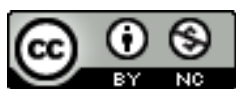

Carnets est mis à disposition selon les termes de la licence Creative Commons - Atribution - Pas d'utilisation commerciale 4.0 International. 


\title{
Arthur Symons traducteur de Baudelaire : D'une traduction partielle à une traduction intégrale
}

\author{
Raphaël Pittier
}

Je tiens à remercier la Professeure Ute Heidmann et le Professeur Jean-Michel Adam pour leur relecture attentive et leurs précieuses suggestions.

1 Pour mieux comprendre le processus qui sous-tend une traduction, il importe de s'intéresser un tant soit peu aux facteurs personnels qui ont orienté le traducteur vers l'auteur qu'il traduit et qui vont orienter à leur tour sa traduction, la conditionner en lui assignant un certain mode de traduire, une finalité spécifique. Cette dimension, qui comprend les divers aspects de la formation du traducteur, gagne notamment à prendre en compte les autres activités littéraires du traducteur pour mieux cerner les éléments qui entrent en dialogue avec l'original.

Dans le cas particulier d'Arthur Symons (1865-1945), il importe premièrement de relever qu'il était lui-même poète ; ce fait peut paraître anodin, mais il se révèle d'une importance capitale si l'on se fie à ceux qui stipulent que «seul un poète peut traduire un poète ". Selon le site Poetry Foundation ${ }^{1}$, il aurait toutefois cessé son activité poétique en 1908 après une dépression nerveuse dévastatrice, mais cette activité l'avait poussé à fréquenter auparavant le célèbre Rhymers' Club (et notamment W. B. Yeats) et on peut penser que cette expérience s'est révélée cruciale pour sa propre production poétique, par un double rapport d'appartenance et de démarcation vis-à-vis de ce groupe littéraire qui place l'auteur dans un interdiscours de la poésie londonienne de la fin du $\mathrm{xix}^{\mathrm{e}}$ siècle. Il faut aussi mentionner une anthologie de la poésie anglaise du xvi ${ }^{\mathrm{e}}$ siècle, et des essais critiques sur Robert Browning et William Blake, entre autres. Enfin, la prise en compte des autres auteurs français traduits par Symons (notamment Stéphane Mallarmé, Paul Verlaine et Émile Verhaeren pour ce qui est des poètes) fournit également des informations primordiales pour mieux cerner le "dialogisme intertextuel et interdiscursif $»^{2}$ qui sous-tend son activité traductoriale. Cela rejoint ce 
que disait Henri Meschonnic sur la composante (du moins partiellement) personnelle de la traduction :

C'est donc autant sur ses propres idées du langage que sur le texte que doit travailler le traducteur. C'est elles qu'il inscrit dans sa traduction autant, sinon plus, que sa compréhension du texte. C'est elles, à travers sa traduction, qu'on voit d'abord. Plus il les cache ou refuse de les voir, plus il les montre. Elles constituent un milieu métalittéraire et métalinguistique qui s'interpose entre le texte et la traduction. Ce milieu composite, mal connu, mal maîtrisé, est le goût, la culture, la situation du traducteur. (Meschonnic, 2012 : 124 ; je souligne)

Les éléments péritextuels (titre, maison d'édition, collection, avertissement, préface, postface, quatrième de couverture) fournissent également de précieuses informations, souvent liées ou reliables à la poétique du traduire sous-jacente, éléments qui ne sauraient être négligés dans l'optique d'une compréhension globale du fait littéraire. Le péritexte recèle des éléments de compréhension supplémentaires quant à la formation générale du traducteur, comme nous allons le voir, et fournit de ce fait une porte d'entrée pour aborder le texte qu'il délimite. C'est par ce biais que nous avons choisi d'aborder le cas particulier des traductions de Baudelaire par Arthur Symons.

\section{Une traduction partielle d'abord}

4 Si les poèmes en vers de Baudelaire ont connu un rapide engouement auprès des traducteurs anglais avec des traductions partielles dès $1869^{3}$, il n'en va pas de même pour ses poèmes en prose, dont la première traduction anglaise, partielle (huit poèmes) par Stuart Merrill, n'apparaît qu'en 1890, dans l'ouvrage Pastels in Prose (Merrill, 1890 : 161-181). Dans cet ouvrage, les poèmes de Baudelaire apparaissent en relation cotextuelle avec les textes de nombreux autres auteurs francophones, rassemblés par Merrill selon une double composante linguistique et générique.

5 En 1905, Arthur Symons signe, chez l'éditeur Elkin Mathews, la première traduction anglaise en volume autonome des poèmes en prose de Baudelaire (Voir Schellino, 2015 : 32), intitulée Poems in Prose, le critère de la taille de ces poèmes disparaissant de la traduction. Mais il s'agit d'une traduction partielle du recueil de Baudelaire tel qu'organisé par Banville et Asselineau: seuls douze poèmes prennent place dans ce petit ouvrage, dont, assez étonnamment, l'épilogue en vers : 


\section{CONTENTS}

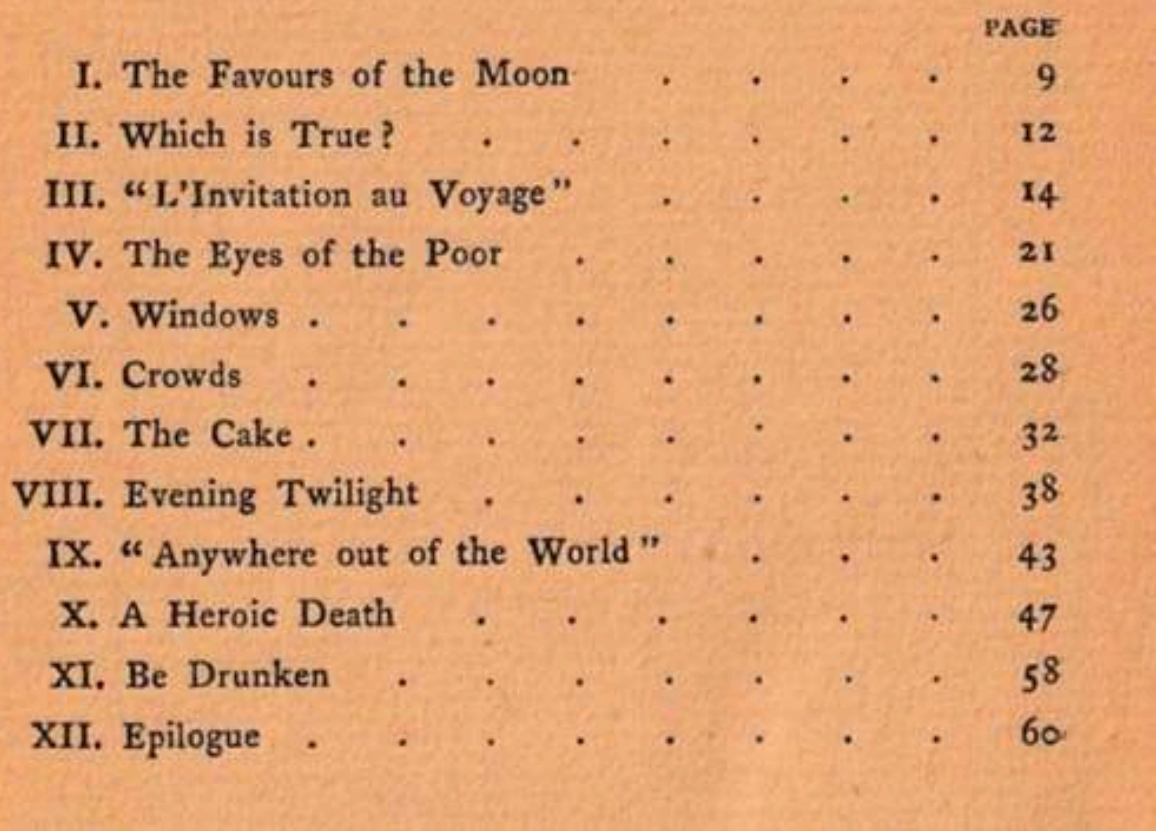

6 À première vue, la sélection et l'ordonnancement des poèmes ne semblent pas répondre à une logique précise, mais le fait de commencer par «The Favours of the Moon » («Les Bienfaits de la Lune ») nous paraît particulièrement intéressant, car, par sa nature, ce conte étiologique de la mélancolie permet de placer d'emblée le lecteur dans un cadre spleenétique. En revanche, une réserve peut être émise quant à la présence de l'épilogue en vers qui introduit une hétérogénéité formelle, d'autant plus qu'il est précédé de chiffres romains, et ainsi apparenté aux poèmes qui le précèdent, alors que, dans le recueil de Baudelaire, il s'en dégageait par l'absence de numérotation. Il est intéressant de constater que ce poème en vers est directement précédé par « Be Drunken ", un des poèmes les plus rythmés du recueil et véritable déclaration de poétique abordant les moyens de surmonter la mélancolie ${ }^{5}$, qui fait en quelque sorte transition vers cet épilogue versifié. L'usage des guillemets se justifie pour le titre du neuvième poème ("Anywhere out of the World") car il portait, à peu de chose près, le même titre ${ }^{6}$ dans l'édition posthume de 1869 , mais il était sous-titré « N'importe où hors du monde ", ce qui conférait à ce poème une dimension bilingue qu'on ne retrouve plus ici. En ce qui concerne le troisième poème ("L'Invitation au Voyage"), les guillemets et le maintien du français peuvent en partie s'expliquer par un renvoi autonymique au texte du poème : "A musician has written an "Invitation à la Valse" : who will compose the "Invitation au Voyage" that we can offer to the beloved, to the chosen sister?» $(1905: 16)^{7}$.

7 Cette table des poèmes est précédée par ce qui nous semble être une préfaceavertissement du traducteur : 
THE "Petits Poèmes en Prose " are experiments, and they are also confessions. "Who of us," says Baudelaire in his dedicatory preface, " has not dreamed, in moments of ambition, of the miracle of a poetic prose, musical without rhythm and without rhyme, subtle and staccato enough to follow the lyric motions of the soul, the wavering outlines of meditation, the sudden starts of the conscience?" This miracle he has achieved in these bagatelles laborieuses, to use his own words, these astonishing trifles, in which the art is not more novel, precise and perfect than the quality of thought and of emotion.' In translating into English a few of these little masterpieces, which have given me so much delight for so many years, I have tried to be absolutely faithful to the sense, the words, and the rhythm of the original.

A. $\mathrm{S}$.

8 Dans cet élément péritextuel, Arthur Symons pointe tout d'abord la dimension expérimentale des poèmes en prose de Baudelaire ; et, de fait, il s'agit véritablement, au plus haut degré, de ce qu'Ute Heidmann identifie comme une expérimentation générique ${ }^{8}$. Puis il reprend, en la traduisant, une phrase de la préface-dédicace à Arsène Houssaye ${ }^{9}$ qui met l'accent sur les particularités de cette prose; en choisissant de traduire " heurtée » par le terme italien "staccato », le traducteur renforce encore la musicalité évoquée dans ces lignes, qui apparait alors comme une condition nécessaire à cette pratique générique ${ }^{10}$.

9 La phrase suivante, qui reprend, doublement modalisée en discours second par l'emploi des italiques et la mention de la source, l'expression «bagatelles laborieuses » que Baudelaire avait utilisée pour désigner ces poèmes en prose dans sa lettre à SainteBeuve du 4 mai 1865. Symons parle d'un miracle accompli par l'auteur ("This miracle he has achieved ») et nous sommes bien d'accord avec lui, mais il ne faut pas oublier que, dans cette même préface-dédicace "À Arsène Houssaye ", Baudelaire met en place une scénographie en trompe-l'œil qui est plutôt celle du demi-échec quant à ses intentions initiales ${ }^{11}$ :

Mais, pour dire le vrai, je crains que ma jalousie ne m'ait pas porté bonheur. Sitôt que j'eus commencé le travail, je m'aperçus que non-seulement je restais bien loin de mon mystérieux et brillant modèle [Aloysius Bertrand], mais encore que je faisais quelque chose (si cela peut s'appeler quelque chose) de singulièrement différent, accident dont tout autre que moi s'enorgueillirait sans doute, mais qui ne peut qu'humilier profondément un esprit qui regarde comme le plus grand honneur du poëte d'accomplir juste ce qu'il a projeté de faire. (Baudelaire, $1869: 2-3$ )

Symons énumère les multiples qualités des poèmes en prose de Baudelaire. On est clairement dans le registre de l'épidictique, et les nombreuses louanges adressées par le traducteur légitiment sa déclaration d'intention finale, celle de rester le plus fidèle 
possible aux aspects de la textualité et de la discursivité des Petits Poèmes en prose que le traducteur juge essentiels: "In translating into English a few of these little masterpieces (...), I have tried to be absolutely faithful to the sense, the words, and the rhythm of the original. " (je souligne) Ce dernier énoncé permet de sceller un pacte entre le traducteur et son lecteur et, de fait, les composantes jugées essentielles sont relativement bien respectées dans cette traduction ${ }^{12}$.

11 L'édition de 1908, publiée par Thomas B Mosher, reprend les mêmes traductions des mêmes poèmes, dans le même ordre. De très rares transformations peuvent néanmoins être relevées au niveau microtextuel. Par exemple, dans l'avertissement que nous venons de parcourir, à la première ligne, l'orthographe ancienne du mot « Poëmes », chère à Baudelaire, est rétablie. Des transformations peuvent également être pointées au niveau typographique, avec l'apparition de lettrines et de cul-de-lampe, mais la différence majeure réside dans l'ajout d'un «Foreword» de l'éditeur, qui mentionne une traduction antérieure des poèmes en prose de Baudelaire par Lafcadio Hearn, et, en note, la traduction partielle de Stuart Merrill que nous avons déjà évoquée. Au niveau de l'intertextualité, l'éditeur fait également un rapprochement très pertinent entre «Les Bienfaits de la Lune » et le poème en vers "The Moon's Minion » (1885) d'Andrew Lang, qui paraît dans la section «Verses and Translations » et qui est donc, malgré un « remaniement radical ${ }^{13}$ dû au régime poétique différent, une véritable traduction du poème en prose de Baudelaire ${ }^{14}$. Cette relation intertextuelle semble fournir une seconde raison présidant au placement des « Favours of the Moon » en tête des poèmes traduits par Symons pour ces éditions partielles. La réimpression de l'édition de 1905, chez Elkin Mathews, en 1913, n'a guère d'autre intérêt que la mise en collection (au sein de la collection « The Vigo Cabinet Series »), qui est une opération éditoriale.

\section{La traduction intégrale}

En 1925 paraît, édité par la Casanova Society, un ouvrage qui propose non seulement une traduction de l'ensemble des Petits Poèmes en prose par Arthur Symons, mais qui place cette traduction en relation cotextuelle avec celles des Fleurs du Mal, et des Paradis artificiels, réalisées par le même traducteur: 




13 Ces textes sont précédés d'une préface du traducteur où l'on voit apparaître, au travers de nombreux patronymes (Rossetti, Catulle, Verlaine, Villon, Poe, Blake, De Quincey, Gautier), une partie du dialogue intertextuel qui sous-tend la traduction à des degrés divers. Par un réseau de comparaisons et de mises en relation, Symons situe Baudelaire par rapport à ces auteurs. Voyons, pour exemple, ce qui rapproche Baudelaire de Catulle selon Symons :

Baudelaire paints vice and the degradation of the lowest depths with cynicism and with pity, as in Une Charogne, where the cult of the corpse is the sensuality of ascetism, or the ascetism of sensuality: the mania of fakirs; material by passion, Catholic by perversity. So, in a sense, he is our modern Catullus. (1925: II)

14 Ces éléments de comparaison, qui prennent ici la forme réduite d'une partie de préface, avaient trouvé une expansion autrement plus conséquente dans la longue étude qu'Arthur Symons consacre à Baudelaire cinq ans auparavant (Symons, 1920) et dans laquelle il se montre un comparatiste aguerri, d'une érudition rare.

Lorsqu'il évoque l'œuvre de Baudelaire, Symons se montre à nouveau dithyrambique, et ne passe pas sous silence l'effet que les poèmes de Baudelaire ont pu avoir sur sa propre activité poétique :

Why did I translate Les Fleurs du Mal ? Because, for one thing, that book of his, in regard to my earliest verses, was at once a fascination and an influence, and because from that time onwards his fascination has been like a spell to me, and because that masterpiece has rarely, if ever, been equalled, has rarely, if ever, been surpassed. (1925: I)

Symons met aussi en évidence le génie de Baudelaire en tant que traducteur (de Poe) et en tant que critique d'art (Wagner, Delacroix, Manet, Constantin Guys). Il est intéressant de constater qu'Arthur Symons partage avec Baudelaire la plupart de ces centres d'intérêt. 


\section{Structure de l'édition de 1925} Society en 1925 : «CHARLES BAUDELAIRE. "Les fleurs du mal, Petits poèmes en prose, Les paradis artificiels." Translated by ARTHUR SYMONS ", l'ouvrage débute avec la traduction des poèmes en prose, dont les titres, à l'exception de "L'invitation au voyage " et de "Mademoiselle Bistouri ", sont traduits en anglais. Nous avons déjà tenté d'expliquer pourquoi le premier reste en français en vertu d'une modalisation autonymique qui fait référence à une œuvre musicale (fictive) contenue dans le corps du poème; pour le second, il faut sans doute y voir le respect d'un patronyme et du titre qui l'accompagne et qui souligne le caractère fondamentalement français de ce personnage ${ }^{15}$. Les poèmes en prose retrouvent leur ordre "original ", c'est-à-dire l'ordre qui était le leur dans le tome IV de l'édition des Euvres complètes ${ }^{16}$ de Baudelaire, paru chez Michel Lévy en 1869 pour ce tome qui contient également, cotextuellement, Les Paradis artificiels, ainsi que (mais absents de la page de titre) La Fanfarlo et Le Jeune Enchanteur. La comparaison des différentes éditions des traductions de Symons montre que le traducteur n'a pas retravaillé les traductions déjà effectuées pour leur intégration à l'édition de $1925^{17}$. En ce qui concerne les titres des poèmes, outre la suppression des guillemets pour "L'invitation au voyage " et «Anywhere out of the world» dont nous avons déjà brièvement parlé, il faut remarquer que tous les titres, dans l'édition de 1925, sont suivis d'un ponctuant. Il s'agit majoritairement d'un point ; seuls trois titres font exception avec un ponctuant plus expressif: "Already!», «Which is true ? " et « Knock down the poor!», comme c'était le cas pour l'édition française de 1869.

Mais, en ce qui concerne le péritexte des Petits poèmes en prose de cette édition de 1925, il y a un élément qui semble éclipser tous les autres tant il est surprenant: c'est l'omission de l'épitre dédicatoire "À Arsène Houssaye" qui a depuis acquis (certainement à tort) ${ }^{18}$ un statut de préface au recueil dans la majorité des éditions ${ }^{19}$. Or, Arthur Symons était, bien évidemment, au courant de ce texte qui précède les poèmes en prose de Baudelaire dans l'édition posthume de 1869 ; c'est même de là que vient la deuxième phrase de son avertissement, explicitement restituée à l'endroit précis de son emprunt: " "Who of us," says Baudelaire in his dedicatory preface, "has not dreamed, in moments of ambition, of the miracle of a poetic prose, musical without rhythm and without rhyme, subtle and staccato enough to follow the lyric motions of the soul, the wavering outlines of meditation, the sudden starts of the conscience ?" ». Si l'on peut encore aisément concevoir qu'il ait décidé d'écarter ce texte de l'édition partielle (bien qu'ayant par ailleurs décidé d'y faire figurer l'épilogue en vers), cette épitre dédicatoire brille par son absence dans la traduction intégrale de 1925. On pourrait bien sûr penser que Symons écarte volontairement ce texte, ayant compris bien avant les autres que cette épitre dédicatoire n'était que contextuellement liée à la publication de plusieurs séries de poèmes dans La Presse que dirigeait Houssaye, mais cette hypothèse reste assez peu probable, puisqu'elle nécessiterait une connaissance des publications pré-originales (en journaux et revues) des poèmes de Baudelaire de la part du traducteur. Une explication plus rationnelle semble pouvoir être déduite de la cotextualité de l'édition de 1925. En effet, rappelons-le : contrairement à l'ordre des textes de Baudelaire annoncé sur la page de titre, l'ouvrage s'ouvre, après une assez 
longue préface d'Arthur Symons, sur Les petits poèmes en prose. Or la préface en question reprend, dans son paragraphe conclusif, l'avertissement qui précédait les poèmes dans l'édition de 1905 (cf. Fig.2). Par la traduction partielle, dans son avertissement, puis dans sa préface, de la lettre-préface "À Arsène Houssaye », Symons en sélectionne les éléments qui sont selon lui les plus importants, les plus dignes d'intérêt, et passe sous silence ceux qui ne méritent pas d'être exposés car ils desservent l'auteur plus qu'ils ne lui rendent gloire. Ainsi l'allusion à Aloysius Bertrand, qui relève de la prétérition, estelle écartée, et surtout la scénographie en trompe-l'œil de l'échec (ou du demi-échec) mise en place par Baudelaire en ayant pris comme objectif l'imitation de ce poète. Quoi qu'il en soit, en terminant sa préface sur ce paragraphe conclusif, la transition se fait naturellement vers les poèmes en prose, grâce à une configuration cotextuelle qui permet l'éviction de l'épitre dédicatoire "À Arsène Houssaye ", qui aurait terni l'admiration que Symons voue à Baudelaire. ${ }^{20}$

\section{La traduction des Fleurs du Mal}

Toujours dans sa préface à l'édition de 1925, Symons ne tarit pas d'éloges sur toute l'œuvre de Baudelaire, de ses poèmes en vers à ses traductions de Poe, en passant par son essai sur les Paradis artificiels, sans omettre non plus ses critiques d'art, montrant ainsi les contours de ses multiples facettes :

Baudelaire, who was infallible, spent most of his life in writing one book of verse (out of which all French poetry has come since his time) Les Fleurs du Mal, 1857; one book of prose, in which prose becomes a fine art - Les Paradis Artificiels, 1860, some criticism which is the sanest, subtlest, and surest which his generation produced, and a translation which is better than a marvellous original. I allude, of course, to his translations of Poe's Prose. (1925 : II)

Cette admiration sans bornes, marquée par les superlatifs, ne semble jamais feinte. L'éloge ne concerne pas que Les Fleurs du Mal, comme c'est trop souvent le cas, mais tous les pans du génie de Baudelaire; et, à propos de ses traductions de Poe, Symons dit quelque chose d'essentiel: qu'une traduction peut être meilleure qu'un merveilleux original, assertion qui n'est pas pour nous déplaire, car une telle déclaration va dans le sens d'une auctorialité traductoriale.

Pour sa traduction des Fleurs du Mal, Arthur Symons se base sur la première édition, celle de 1857, dont six pièces furent condamnées pour « outrage à la morale publique et aux bonnes mœurs ». Et en effet, c'est d'un Baudelaire sulfureux que Symons fait le portrait lorsqu'il dépeint l'auteur des Fleurs du Mal en le comparant à un Catulle moderne, dont la poésie contient « a deliberate science of sensual and sexual perversity which has something curious in his accentuation of vice with horror " $(1925: \mathrm{II})^{21}$. On peut concevoir que le Baudelaire des Fleurs du Mal (et surtout des Fleurs condamnées de la première édition) puisse être ainsi représenté par certains esprits manichéens, mais il ne saurait, à notre avis, se laisser réduire à cela. La préface de Symons, dont ces quelques lignes ont peut-être été dictées par la volonté d'aller dans le sens d'une certaine ligne éditoriale, ou de répondre aux envies d'un certain lectorat cherchant à perpétuer, voire à renforcer, cette représentation de Baudelaire, est la trace d'un dialogue intertextuel traductorial qui participe de la réception quelque peu calamiteuse de l'auteur dans les pays anglo-saxons. 
En se basant sur la première édition des Fleurs du Mal, Symons se permet l'audace de faire paraître des (traductions de) textes condamnés et interdits en France. De fait, la réhabilitation des pièces condamnées des Fleurs du Mal ne surviendra qu'en 1949; entre-temps, les éditeurs qui voulaient publier ces pièces (dont Auguste PouletMalassis, l'éditeur de Baudelaire) étaient obligés de le faire à l'étranger (en Belgique notamment). Les traductions de ces poèmes n'étaient apparemment pas touchées par cette interdiction, du moins celles qui n'étaient pas publiées sur le territoire français.

Contrairement aux poèmes en prose, les poèmes des Fleurs $d u$ Mal, dans cette traduction d'Arthur Symons, gardent leur titre original : en français pour la plupart, en latin pour quatre d'entre eux ("Sed non satiata», "De profundis clamavi », "Franciscae meae laudis » et «Moesta et errabunda ») et neuf poèmes qui ne sont désignés que par leur numéro en chiffres latins. Le poème liminaire « Au lecteur » est conservé sans surprise. Nous n'avons guère d'explication convaincante à proposer pour cette non-traduction des titres, surtout après la traduction des titres des poèmes en prose. Peut-être est-ce dû à la présence de titres en latin ; peut-être est-ce la marque d'un régime de poéticité différent, lié à une inscription d'énoncés dans deux pratiques génériques distinctes ; peut-être encore faut-il y voir la volonté de hiérarchiser, comme souvent, le vers et la prose...

\section{Traduction des Paradis artificiels}

Si Arthur Symons se montre extrêmement élogieux envers l'œuvre de Baudelaire dans son ensemble, il semble éprouver pour Les Paradis artificiels un intérêt encore supérieur, comme il l'écrit dans sa préface :

Les Paradis Artificiels: Opium and Haschisch is the most wonderful book Baudelaire ever wrote. It has this astonishing logic which he possessed supremely, which unravels, with infinite precautions, every spider's web of this seductive drug, which enslaves the imagination, which subdues the will, which turns sounds into colours; which annihilates time and space; which to Baudelaire, and to those who, like myself, have undergone its fascination, has the occult divinity of an insidious and mysterious mistress. (1925: Iv)

On le voit, le traducteur insiste ici plus sur la fonction pratique (voire pragmatique) de cet essai que sur sa dimension esthétique. Indépendamment de cette remarque, Les Paradis artificiels, troisième volet de cette traduction de Baudelaire par Arthur Symons, constituent un cas d'étude intéressant. Il s'agit là aussi d'une traduction partielle, puisque, dans le tome IV des Euvres complètes de Baudelaire, où il apparaît en cotexte aval des Petits Poëmes en prose, il est composé de trois parties (en plus de la dédicace) : Le Poëme du Haschisch; Un Mangeur d'opium; et Du Vin et du Haschisch, comparés comme moyen de multiplication de l'individualité. Une version réduite, amputée notamment de la troisième partie, parait chez Poulet-Malassis en 1860. C'est apparemment l'édition que cite le traducteur (voir supra). Quoi qu'il en soit, Arthur Symons ne traduit que la partie « Le Poëme du Haschisch », et renonce, volontairement, à traduire « Un Mangeur d'opium ».

On pourrait tout d'abord croire que, si le traducteur renonce à traduire « Un Mangeur d'opium ", c'est parce que ce texte est lui-même une traduction-adaptation de Confessions of an English Opium-Eater de Thomas De Quincey par Baudelaire. Retraduire le texte ainsi obtenu pourrait donc, pour Arthur Symons, manquer d'intérêt ${ }^{22}$. Il n'en est 
rien. Baudelaire prend de telles libertés avec le texte de De Quincey, mêlant résumés, digressions, ajouts personnels, que le texte obtenu est radicalement autre, et Symons cite justement à ce propos, dans sa préface, une lettre de Baudelaire à Malassis qui va tout à fait dans ce sens :

De Quincey est un auteur affreusement conversationniste et digressioniste, et ce n'était pas une petite affaire que de donner à ce résumé une forme dramatique et d'y introduire l'ordre. De plus, il s'agissait de fondre mes sensations personnelles avec les opinions de l'auteur original et d'en faire un amalgame dont les parties fussent indiscernables. (Lettre à Auguste Poulet-Malassis du 16 février 1860)

Du type de traduction utilisé par Baudelaire pour traduire le texte de De Quincey résulte donc un texte radicalement différent de l'original, et qui pourrait donc aisément être retraduit vers l'anglais sans risque de lasser le lecteur familier du texte original. Et, de fait, Symons invoque une raison contraire, qu'il explicite également dans sa préface, pour justifier l'absence de traduction d'« Un Mangeur d'opium » :

In translating Les Paradis artificiels I have deliberately omitted those interpolations which Baudelaire introduced into his versions of De Quincey's prose simply for this reason that, in taking The Confessions of an Opium-Eater as his main subject, he took for his foundation the stone which the builders rejected and in thus giving a proof of his own wisdom in building on this shifting foundation an edifice as imperishable as the earth's own self - this masterpiece, which apart from the necessary digressions, remains, in its way, unique in literature. (1925: III)

Arthur Symons, qui se montrait si élogieux et si enthousiaste en ce qui concerne les traductions de Baudelaire de l'œuvre en prose de Poe, montre ici beaucoup plus de réserve quant à sa traduction des Confessions of an English Opium-Eater de De Quincey. Alors que Baudelaire reprochait surtout à ce dernier ses nombreuses digressions et un certain manque d'ordre dans son récit, Symons reproche surtout à Baudelaire ses «interpolations " qui aboutissent à une modification substantielle du texte, à une transformation en profondeur qui affecte la structure compositionnelle du texte original. Cette modification d'ordre compositionnel, souhaitée par Baudelaire et obtenue au prix d'un grand effort, n'est donc pas du goût de Symons pour qui la traduction de Baudelaire ne rend plus guère l'esprit de l'original. En fin de compte, c'est ici la fidélité de la traduction à l'original qui est en question, et on peut comprendre le point de vue d'Arthur Symons, pour qui la traduction idéale devrait se montrer, comme il l'écrit à la fin de son avertissement, « fidèle au sens, aux mots, et au rythme de l'original. » On est effectivement bien loin de cet idéal avec la traduction des Confessions de De Quincey, mais cet éloignement par rapport à l'original, qui suscite la désapprobation de Symons, est tout à fait assumé par Baudelaire ; et, dans ce cas précis, l'auctorialité du traducteur ne peut plus guère être remise en question, puisque l'auteur-traducteur (Baudelaire) produit une œuvre radicalement différente - et certainement meilleure - à partir de l'original qui lui sert de point de départ, y insérant notamment des réflexions et des commentaires méta-narratifs relevant de sa propre expérience.

\section{Baudelaire et Symons : un dialogue privilégié}

Il arrive régulièrement qu'un auteur se sente naturellement porté vers un autre auteur, en raison d'une certaine connivence d'esprit, d'âme, ou de style. Lorsque ce «double littéraire » vient d'une autre culture, écrit dans une autre langue, l'envie de le traduire 
s'impose souvent à l'esprit de son " correspondant ", surtout s'il n'existe pas encore de traduction, ou pas de traduction satisfaisante ${ }^{23}$. Car traduire, c'est s'approprier le texte d'un autre auteur, l'interpréter d'une certaine manière, et enfin le transposer dans sa langue en effectuant des choix linguistiques qui sont propres au traducteur et qui sont liés à sa formation.

Cette admiration, qui va parfois presque jusqu'à l'identification ou la jalousie, Baudelaire l'a ressentie à l'égard de Poe (les témoignages ne manquent pas), beaucoup plus qu'à l'égard de De Quincey (qui suscitait davantage un intérêt de nature topique ou thématique). Et, quelques décennies plus tard, c'est au tour d'Arthur Symons de ressentir pour Baudelaire cette attirance intellectuelle qui orientera son travail tout au long de sa vie. Si l'on reprend la notion de "dialogisme " constitutif de tout texte, abordée en début d'article, c'est pour en préciser un point important, à savoir le degré de prégnance des interdiscours en présence. Il nous semble essentiel d'insister ici sur le fait que, parmi la multitude des interdiscours et intertextes qui dialoguent avec un texte, certains doivent être considérés avec plus d'attention que d'autres, en raison de leur densité supérieure, constituant de véritables faisceaux de relations interdiscursives, qui vont converger pour donner à ce réseau interdiscursif une grande cohérence.

Or, dans le cas que nous avons examiné, le réseau "dialogique» qui unit Arthur Symons à Charles Baudelaire est particulièrement dense : d'une part, les deux auteurs pratiquent de nombreuses activités littéraires communes (poésie, critique d'art, traduction) ; ensuite, Symons s'est intéressé à l'ensemble de l'œuvre de Baudelaire et a écrit une étude conséquente à ce sujet; enfin, Symons ne s'est pas contenté de traduire une œuvre de Baudelaire mais tous ses ouvrages majeurs. Certes, Symons ne s'est pas consacré qu'à Baudelaire, et il faudrait également prendre en compte ses autres traductions et autres articles critiques pour mieux cerner les autres réseaux interdiscursifs ${ }^{24}$, mais l'intérêt et l'admiration qu'il a portés à Baudelaire tout au long de sa vie ne peuvent pas l'avoir laissé indemne de tout influence.

Sans entrer véritablement dans les traductions à proprement parler, nous avons vu dans cet article comment nous pouvions dégager des divers éléments péritextuels des informations importantes pour cerner le regard que porte le traducteur sur l'auteur et sur l'œuvre qu'il traduit, sur l'activité du traducteur en général, sur la tâche qu'il se fixe, sur les critères qu'il souhaite respecter en priorité dans sa traduction. En commençant par une traduction partielle des Petits Poëmes en prose, Symons a opéré une sélection de onze poèmes sur les cinquante (l'épilogue en sus) du recueil. Le choix des poèmes correspond sans doute au goût du traducteur; ils ne semblent pas suivre un ordre logique précis (mis à part l'épilogue à la fin). Le fait que le petit recueil recomposé par Symons s'ouvre par "The Favours of the Moon" est tout de même intéressant à nos yeux, puisque ce poème, en tant que conte étiologique de la mélancolie, pourrait être l'emblème du recueil, qui d'une certaine manière l'incarne et le résume. De même, la place assignée au poème «Be Drunken ", juste avant l'épilogue en vers, est intéressante, car il s'agit sans doute du poème le plus rythmé du recueil, et il assurerait ainsi, en quelque sorte, une transition vers le seul poème en vers du recueil.

La traduction intégrale du recueil paraît vingt ans après la première traduction partielle, en 1925, avec, comme cotexte amont, une précieuse préface du traducteur, et, en cotexte aval, une traduction des Fleurs du Mal (dans leur édition censurée de 1857) et 
des Paradis artificiels (amputés de la section «Un Mangeur d'opium " pour des raisons que le traducteur explicite dans sa préface). Entre-temps, Symons avait publié, en 1920, une longue étude sur Baudelaire. Avec Arthur Symons, nous avons donc affaire à un traducteur averti et aguerri, passionné, spécialiste de poésie, qui a également traduit d'autres poètes français, qui connaît extrêmement bien l'œuvre qu'il traduit et la langue qu'il traduit. Ainsi, par analogie à la notion de "lecteur-modèle » développée par Umberto Eco, il semble que nous ayons affaire avec Arthur Symons, à quelqu'un qui se rapproche, de par cette formation plurielle, autant que possible du «traducteurmodèle ».

\section{BIBLIOGRAPHIE}

\section{Corpus}

BAUDELAIRE, Charles (1869). Petits Poëmes en prose. Les Paradis artificiels. Paris : Michel Lévy.

BAUDELAIRE, Charles (1905). Poems in prose (trad. Arthur Symons). London: Matthew Elkins. (réimpression 1913).

BAUDELAIRE, Charles (1909). Poems in prose (trad. Arthur Symons). Portland : Thomas B Mosher. BAUDELAIRE, Charles (1925). Les Fleurs du mal, Petits poèmes en prose, Les Paradis artificiels (trad. Arthur Symons). London: The Casanova Society.

BAUDELAIRE, Charles (1989). Volume II: The Poems in Prose and La Fanfarlo (trad. Francis Scarfe). London : Anvil Editions. BAUdelaire, Charles (2017). Le Spleen de Paris (éd. Aurélia Cervoni \& Andrea Schellino). Paris : GF Flammarion.

\section{Etudes}

ADAM, Jean-Michel (2018). Souvent textes varient. Génétique, intertextualité, édition et traduction. Paris : Classiques Garnier (coll. « Investigations stylistiques », $\mathrm{n}^{\circ} 6$ ).

CHARAUDEAU, Patrick (2015). « Le maelstrom de l'interdiscours », in L'analyse de discours. Sa place dans les sciences du langage et de la communication. Rennes : Presses Universitaires de Rennes. CHARAUDEAU, Patrick \& Maingueneau, Dominique (dir.) (2002). Dictionnaire d'analyse du discours. Paris : Seuil

CLARK, Carol \& Sykes, Robert (éd.) (1997). Baudelaire in English. Londres : Penguin.

COSTE, Bénédicte (2021). "Arthur Symons the translator: Translation, remediation, recognition », in Parallèles. Genève : Université de Genève. [on-line] URL : https://www.paralleles.unige.ch/ files/7016/2188/8211/Paralleles-33-2_Coste.pdf (consulté le 11 octobre 2021) 
DASKALOVA, Yarmila Nikolova (2018). Literary Pairs in Comparative Readings across National and Cultural Divides. Newcastle Upon Tyne : Cambridge Scholars Publishing.

ECO, Umberto (2006). Dire presque la même chose. Expériences de traduction. Paris : Grasset (Le Livre de Poche) (coll. « biblio essais »).

HEIDMANN, Ute (2013). « “C'est par la différence que fonctionne la relation avec un grand R". Pour une approche comparative et différentielle du traduire ", in G. Chiurazzi (éd.), The Frontiers of the Other. Ethics and Politics of Translation. Wien-Zürich-Berlin- Münster: Lit. Verlag, pp. 61-73.

HEIDMANN, Ute (2017). «Que veut et que fait une comparaison différentielle ? Propos recueillis par Jean-Michel Adam \& David Martens ", in Interférences littéraires/Literaire interferencies, $\mathrm{n}^{\circ} 21$, pp. 199-225.

LANG, Andrew, «The Moon's Minion », in Gutenberg Books. [on-line] URL : http://gutenbergbooks.xyz/ balladesandrhymesfromballadesinbluechinaandrhymesalamodebyandrewlang/chapter/the-moonsminion/ (consulté le 26 juillet 2021).

MERRILL, Stuart (1890). Pastels in Prose. New York: Harper \& Brothers, pp. 161-181.

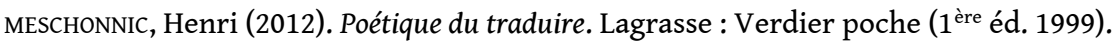

« Arthur Symons ». Chicago: Poetry Foundation [on-line] URL: https://www.poetryfoundation.org/ poets/arthur-symons

SCHELLINo, Andrea (2015). Bibliographie du Spleen de Paris (1855-2014). Paris : Classiques Garnier (coll. « Baudelaire », $\mathrm{n}^{\circ} 1$ ).

symons, Arthur (1920). Charles Baudelaire: A study. New York: E.P. Dutton \& Co.

\section{NOTES}

1. Voir https://www.poetryfoundation.org/poets/arthur-symons (consulté le 12 juin 2021).

2. Ce concept-clé de l'analyse du discours, issu du cercle de Bakhtine, permet de formuler une propriété constitutive de tout texte : celle de répondre à des (inter)textes et des (inter)discours antérieurs ainsi que d'anticiper des réponses potentielles de textes et de discours à venir. Voir, à ce sujet, Charaudeau \& Maingueneau (2002 : 175-178).

3. Voir Coste $(2021: 10)$ pour un historique des premières traductions anglaises de l'œuvre en vers de Baudelaire.

4. Nous indiquons ci-après les titres originaux de ces poèmes, ainsi que, entre parenthèses, leur place dans le recueil de Baudelaire: I. Les Bienfaits de la Lune (xxxvII) ; II. Laquelle est la vraie (xxXVIII) ; III. L'Invitation au Voyage (XVIII) ; IV. Les Yeux des pauvres (xxVI) ; v. Les Fenêtres (xxxv) ; vI. Les Foules (XXII) ; VII. Le Gâteau (xv) ; VIII. Le Crépuscule du soir (XXII) ; IX. Any Where out of the World. N'importe où hors du monde (xLVII) ; x. Une Mort héroïque (xxVII) ; XI. Enivrezvous (XXXIII) ; XII. Epilogue (-).

5. Voir mon article pour Letra Magna pour une analyse des rythmes présents dans ce poème: http://www.letramagna.com/artigos_27/Artigo\%2013.pdf

6. Baudelaire écrit « Any Where » en deux mots.

7. Les guillemets disparaîtront pour les deux titres dans la traduction intégrale de 1925.

8. Pour une définition de ce concept, je renvoie à Heidmann $(2017: 221)$.

9. Jean-Michel Adam (2018: 337) a montré que cette épitre dédicatoire était contextuellement liée à la publication de séries de poèmes dans La Presse et que cette "préface » devrait ainsi être écartée du recueil. 
10. Suivant la proposition d'Ute Heidmann (2017 : 220), il nous semble plus prudent d'écarter la notion trop figée de "genre littéraire" pour envisager la généricité du texte comme une inscription d'énoncés au sein d'une " configuration de pratiques et de formes génériques ».

11. Si cette scénographie semble ironique au lecteur d'aujourd'hui, il n'est pas certain qu'elle l'ait été pour Baudelaire et pour le lecteur contemporain, car Aloysius Bertrand, qui a été entre-temps relégué au rang de poète mineur, jouissait alors d'un important prestige. Il semble par conséquent difficile de déterminer si l'ethos de modestie, et l'éloge du modèle pour se placer sous son égide, sont ici feints ou véritablement assumés par Baudelaire.

12. L'exemple de la traduction du poème "Enivrez-vous ", que j'ai traité de manière plus approfondie en vue de ma thèse de doctorat sur les traductions des Petits Poèmes en prose de Baudelaire en anglais, allemand, italien et espagnol, laisse toutefois entrevoir, au niveau sémantique, des différences importantes dues à l'« impossible synonymie » (Heidmann, 2013 : 62) entre les langues et, au niveau rythmique, des différences inéluctables liées aux structures syntaxiques et rythmiques de ces langues.

13. On peut, avec Umberto Eco, parler de «remaniement radical " pour définir ce type de "phénomène qui, du point de vue éditorial et commercial, entrerait dans la catégorie de la traduction proprement dite et qui représente pourtant en même temps un exemple flagrant de licence interprétative » (2006:378).

14. Le site Gutenberg Books signale du reste, entre le titre et le poème: "(From the prose of $C$. Baudelaire.) » (Lien dans la bibliographie)

15. Par la suite, de nombreux traducteurs choisiront de traduire ce titre en anglais, comme par exemple Francis Scarfe qui donne pour titre de ce poème (dans une édition avec testo a fronte) « Miss Lancet » (cf. Baudelaire, 1989 : 225)

16. L'intervention de Théodore de Banville et Charles Asselineau, intervenant en tant qu'«instance énonciative de transmetteur supplétif de l'auteur» (Adam, 2018: 332) a été critiquée à maints égards, mais guère au sujet de l'ordre des poèmes du recueil, puisqu'ils se sont basés, pour ce faire, sur une liste établie par Baudelaire lui-même.

17. Les rares transformations sont situées au niveau microtextuel et semblent être davantage des décisions (ou des coquilles) imputables à l'éditeur ou au typographe qu'à une véritable intention de l'auteur-traducteur. Par exemple, dans «Which is True ? ", Symons traduit « (...) et c'est moimême qui l'ai enterrée, un jour que le printemps agitait son encensoir jusque dans les cimetières » par « (...) and I buried her with my own hands, one day when Spring shook out her censer in the graveyards. (1905: 12; 1909: 7-8), alors que l'édition de 1925 singularise étrangement le dernier substantif : « (...) graveyard. » (1925:68).

18. Voir Adam (2018 : 337).

19. L'édition du Spleen de Paris d'Aurélia Cervoni et Andrea Schellino (GF Flammarion, 2017) fait en ce sens office de pionnier en écartant délibérément ce texte du recueil.

20. Les poèmes traduits pour l'édition partielle de 1905 n'ayant pas passé par une phase de récriture (ou de retraduction) en vue de leur intégration au sein de la traduction intégrale du recueil, il serait intéressant d'en observer la cohérence au niveau de l'intratextualité (réseaux et récurrences de vocables, traitement des relations intratextuelles privilégiées) et de la cotextualité (re-sémantisation différente des poèmes - provisoirement dégagés de leurs liens cotextuels initiaux - liée à leur réintégration à la configuration originale du recueil, liée à leur réintégration au sein de leur structure signifiante), mais une telle étude dépasse le cadre de cet article et je traiterai ce phénomène de manière plus approfondie dans ma thèse de doctorat.

21. Bénédicte Coste (2021 : 12-13) met en lumière la représentation d'un Baudelaire sataniste que pouvait se faire Symons.

22. Nous savons à quel point le fait de retraduire dans la langue d'origine peut s'avérer fécond d'un point de vue heuristique, mais ce point de vue n'est malheureusement pas partagé par tout le monde, notamment par les traducteurs. 
23. Le cas de Baudelaire traduisant les œuvres en prose d'Edgar Poe est un bon exemple de ce type de transfert culturel. Pour un développement des éléments qui favorisent le rapprochement de cette " paire littéraire », voir Daskalova (2018: 24-47).

24. L'image du «maelstrom de l'interdiscours » utilisée par Patrick Charaudeau (2015 : 125-126) permet de rendre compte des différents réseaux interdiscursifs qui sous-tendent l'œuvre. Bien qu'à la base, il s'apparente plutôt à un phénomène auctorial, nous sommes persuadés qu'un tel «maelstrom interdiscursif » s'applique également à l'opération de traduction, et d'autant plus si l'on considère l'acte traductorial comme une récriture à part entière.

\section{RÉSUMÉS}

Cet article traite des traductions de Charles Baudelaire effectuées par Arthur Symons. Il s'agit tout d'abord d'une traduction partielle des Petits Poèmes en prose, parue en 1905 et en 1908, puis d'une traduction intégrale de ce recueil, parue en 1925 au sein d'un volume où paraissent également, cotextuellement, les traductions des Fleurs du Mal et des Paradis artificiels, réalisées par le même traducteur. La prise en compte du péritexte de ces éditions (tables des matières; avertissement du traducteur ; préface de l'éditeur ; préface du traducteur) permet une première approche de ces traductions, car les commentaires méta-traductoriaux qui y sont contenus permettent de légitimer et de justifier certains choix opérés par le traducteur, ainsi que de mettre à jour tout un réseau interdiscursif qui va sous-tendre la traduction.

This article deals with Arthur Symons' translations of Charles Baudelaire. It first consists of a partial translation of the Petits Poèmes en prose, published in 1905 and 1908, and then of a complete translation of this collection of poems, published in 1925 in a volume in which the translations of Les Fleurs $\mathrm{du}$ Mal and the Paradis artificiels, made by the same translator, also appeared. Taking into account the peritexts of these editions (tables of contents; translator's foreword; editor's preface; translator's preface) allows an original approach of these translations, as the meta-translation comments contained therein make it possible to legitimize and justify certain choices made by the translator, as well as to uncover a whole interdiscursive network that underpins the translation.

\section{INDEX}

Mots-clés : Baudelaire (Charles), Symons (Arthur), traduction, péritextualité, dialogisme

Keywords : Baudelaire (Charles), Symons (Arthur), translation, peritextuality, dialogism

\section{AUTEUR}

\section{RAPHAËL PITTIER}

Université de Lausanne - CLE

Raphael.Pittier[at]unil.ch 